\title{
COMPOSIÇÃO QUÍMICA E COMPOSTOS BIOATIVOS PRESENTES NA POLPA E NA AMÊNDOA DO PEQUI (Caryocar brasiliense, Camb.) ${ }^{1}$
}

\author{
ALESSANDRO DE LIMA², ANA MARA DE OLIVEIRA E SILVA ${ }^{3}$, REGINALDO ALMEIDA TRINDADE 3 , \\ ROSÂNGELA PAVAN TORRES ${ }^{3}$, JORGE MANCINI-FILHO $^{4}$
}

RESUMO - O conhecimento da composição química dos alimentos é fundamental para se avaliarem a disponibilidade de nutrientes e o seu consumo por populações. Neste trabalho, o pequi (Caryocar brasiliense, Camb.) foi caracterizado pela composição centesimal e pela presença de compostos bioativos na polpa e na amêndoa. Os dados do perfil lipídico mostram alto teor de lípides tanto na polpa quanto na amêndoa, destacando-se nos mesmos a presença dos ácidos graxos insaturados, predominando o ácido oléico como principal componente entre os ácidos graxos. Foi observada também a relação entre os elevados teores de ácidos graxos insaturados com os compostos fenólicos e carotenóides presentes, tendo a polpa quantidades mais expressivas dessas substâncias quando comparada à amêndoa, além de conter uma quantidade superior de fibra alimentar. Os resultados obtidos abrem a perspectiva de se utilizar o pequi como fruto que apresenta, na sua composição, compostos importantes para a formulação de uma dieta saudável.

Termos para indexação: pequi, perfil de ácidos graxos, fibras, compostos bioativos.

\section{CHEMICAL COMPOSITION AND BIOACTIVE COMPOUNDS IN THE PULPAND ALMOND OF PEQUI FRUIT}

\begin{abstract}
The knowledge of the chemical composition of foods is basic for evaluate the nutrients availability and its consumption for the population. In this work, the pulp and the almond of pequi fruit (Caryocar brasiliense, Camb.) were characterized by the centesimal composition and the presence of nutrients. The results showed high amount of lipids and in the fatty acids profile, the oleic fatty acid was the main component. The presence between high unsaturated fatty acids and antioxidant compounds (phenolic acids and carotenoids) was correlated with the fruit protection. In the pulp was observed too high amount of alimentary fiber. These results are suggesting the pequi utilization in a healthful diet preparation.
\end{abstract}

Index Terms: pequi, fatty acid profile, fiber, bioactive compounds.

O conhecimento da composição nutricional dos alimentos consumidos no Brasil é fundamental para se avaliarem a disponibilidade de nutrientes e o seu consumo por populações, além de verificar a adequação nutricional da dieta, identificar o estado nutricional, desenvolver pesquisas sobre as relações entre dieta e doença, no planejamento agropecuário e na indústria de alimentos, entre outros. Entretanto, pelas suas dimensões continentais, nosso País possui ainda uma infinidade de alimentos, principalmente de origem vegetal, que devem ser melhor caracterizados (Núcleo de Estudos e Pesquisa em Alimentação, 2004).

O pequi (Caryocar brasiliense, Camb.), conhecido popularmente como piqui, pequiá, amêndoa de espinho, grão de cavalo ou amêndoa do Brasil, é cultivado em todo o cerrado brasileiro, que inclui os Estados do Pará, Mato Grosso, Goiás, Distrito Federal, São Paulo, Minas Gerais e Paraná, como também nos Estados nordestinos, Piauí, Ceará e Maranhão. Sua frutificação ocorre principalmente entre os meses de janeiro a março, podendo ser encontrados frutos fora dessas épocas (Ribeiro, 2000). Esses são constituídos pelo exocarpo ou pericarpo, de coloração esverdeada ou marrom-esverdeada, mesocarpo externo, polpa branca com coloração pardoacinzentada e mesocarpo interno, que constitui a porção comestível do fruto, possuindo coloração amarelada, e separase facilmente do mesocarpo externo quando maduro. $\mathrm{O}$ endocarpo, que é espinhoso, protege a semente ou amêndoa, que é revestida por um tegumento fino e marrom, sendo também uma porção comestível (Melo Junior et al., 2004).

$\mathrm{O}$ fruto de pequi é consumido pelas populações que habitam as regiões onde são produzidos. O Estado de Minas Gerias (MG) é o principal produtor e consumidor do pequi, sendo que, no ano de 2003, foram comercializados aproximadamente $20.000 \mathrm{~kg}$ de pequi. A polpa do pequi é utilizada na elaboração de diferentes pratos, como: arroz com pequi, feijão com pequi, frango

(Trabalho 154-06). Recebido em : 04-10-2006. Aceito para publicação em: 19-07-2007.

${ }^{2}$ Professor do Curso de Tecnologia em Alimentos, CEFET-PI, Doutorando em Ciências de Alimentos FCF-USP. Fone (0XX 1130913688 ). e-mail: alima@usp.br

3 Mestrandos em Ciência dos Alimentos da Faculdade de Ciências Farmacêuticas/USP- São Paulo-SP. Fone (0XX 11 30913688). anamara@usp.br, realtrin@usp.br, rptorres@usp.br

${ }^{4}$ Professor Titular Do Departamento de Alimentos e Nutrição Experimental da Faculdade de Ciências Farmacêuticas/USP- São Paulo-SP. Fone (0XX 11 30913688).jmancini@usp.br

Apoio Financeiro: CNPq 
com pequi, cuscuz com pequi e o tradicional baião de três: arroz, feijão e pequi. Já a amêndoa é utilizada como ingrediente de farofas, doces e paçocas, além de ser consumida salgada como petisco. Por se tratar de um fruto de fácil produção e com características desejáveis em relação ao sabor e valor nutritivo, o pequi pode representar uma fonte potencial na alimentação e sobrevivência de uma parcela da população brasileira (Ribeiro, 2000).

Por outro lado, o consumo de frutas e vegetais tem sido associado a uma menor incidência e mortalidade por diversas doenças crônicas não-transmissíveis. A proteção que esses alimentos oferecem contra as enfermidades degenerativas, como câncer, doenças cardiovasculares e cerebrovasculares, está associada ao seu alto conteúdo de constituintes químicos com propriedades importantes, como as de antioxidantes (vitamina C, E, carotenóides e polifenóis) (Hinneburg et al., 2006).

O objetivo deste trabalho foi caracterizar nutricionalmente o pequi, através da determinação da sua composição centesimal, do perfil de ácidos graxos e dos teores de compostos fenólicos e carotenóides totais na sua polpa e amêndoa.

Os frutos de pequi foram coletados em árvores adultas no solo (dezembro/2004), na zona rural do município de Santa Rosa, pertencente à microrregião de Picos, coordenadas geográficas de $06^{\circ} 45^{\prime} 57^{\prime \prime}$ de latitude sul e $42^{\circ} 17^{\prime} 17^{\prime \prime}$ de longitude oeste de Greenwich, Estado do Piauí - Brasil.

Logo após a colheita, a casca e a polpa dos frutos de pequi foram retiradas com faca, à temperatura ambiente $\left( \pm 23^{\circ} \mathrm{C}\right)$, e a polpa seca, em estufa com ar forçado a $60^{\circ} \mathrm{C}$, por 24 horas. Após a secagem, as amostras foram trituradas em moinho analítico, passadas em peneira de 32 Mesh e armazenadas a $20^{\circ} \mathrm{C}$.

Os caroços foram secos em estufa com ar forçado a $60^{\circ} \mathrm{C}$, por 36 horas, para facilitar a retirada da amêndoa ou semente. Em seguida, as sementes foram retiradas com uma pinça e trituradas conforme procedimento anteriormente descrito.

Com relação à composição centesimal da polpa e da amêndoa, foram determinados: umidade, resíduo mineral fixo (cinzas), proteínas, lipídios totais e fração fibroalimentar total (AOAC, 1998); os carboidratos foram obtidos por diferença; o valor energético total (VET) foi estimado, considerando-se os fatores de conversão de Atawer de $4 \mathrm{Kcal} / \mathrm{g}$ de proteína, $4 \mathrm{Kcal} / \mathrm{g}$ de carboidrato e $9 \mathrm{Kcal} / \mathrm{g}$ de lipídio.

Para a determinação da composição em ácidos graxos, foram realizadas extração e esterificação conforme descrito por Folch et al. (1957) e Hartman \& Lago (1973), respectivamente. Os ácidos graxos foram separados, utilizando-se de um cromatógrafo a gás marca Shimadzu, modelo GC17A, com detector de ionização de chama e coluna capilar de sílica fundida, Carbowax 20M (30 m x 0,25 mm diâmetro interno), utilizando como referência_padrões de ácidos graxos da Sigma.

Os fenólicos totais foram determinados de acordo com Swain \& Hills (1995), e os carotenóides totais foram extraídos e quantificados conforme Almeida \& Penteado, (1988).

Todas as análises foram realizadas em triplicata, e os resultados obtidos foram expressos em média e desvio-padrão entre as amostras.
A polpa do fruto de pequi é rica em lipídios $(33,4 \%)$. Também se constitui em uma fonte importante de fibra alimentar $(10,02 \%)$ e um teor de $3 \%$ de proteínas, fornecendo cerca de $358 \mathrm{Kcal} / 100 \mathrm{~g}$ de material, as quais correspondem a $18 \%$ das necessidades calóricas de um adulto com uma dieta de $2.000 \mathrm{Kcal}$ e $40 \%$ das necessidades de fibra alimentar (Brasil, 2003). Isso mostra que o consumo da polpa de pequi poderá trazer benefícios à saúde da população, tendo em vista o conhecimento de que o consumo regular de fibra alimentar na dieta está relacionado com a redução do risco de diversos quadros patológicos (Michels et al., 2005).

Os quatro componentes majoritários da amêndoa de pequi foram os lipídios $(51,51 \%)$, as proteínas $(25,27 \%)$, os carboidratos $(8,33 \%)$ e a fibra alimentar $(2,2 \%)$, apresentando um baixo teor de umidade e um teor elevado de minerais representado pelas cinzas (Tabela 1).

Tanto na polpa como na amêndoa do pequi, pode ser observado o predomínio dos ácidos graxos insaturados com $61,35 \%$ e $52,17 \%$, respectivamente. O ácido oléico está presente em maior concentração na polpa, com $55,87 \%$, sendo seguido pelo ácido palmítico $(35,17 \%)$. Na amêndoa do pequi, predominam os ácidos palmítico e oléico em quantidades praticamente iguais, $43,76 \%$ e $43,59 \%$, respectivamente. Também estão presentes o ácido linoléico com 5,51\%, esteárico com 2,04\%, palmitoléico com $1,23 \%$, sendo detectado em menores quantidades outros ácidos graxos (Tabela 2). Assim, tanto a polpa como a amêndoa do pequi possuem ácidos graxos importantes para compor uma dieta saudável.

O pequi é um fruto encontrado em regiões onde as árvores recebem alta incidência de raios solares, o que favorece a geração de radicais livres, além do que, tanto a polpa quanto a amêndoa do pequi são ricas em lipídios (Tabelas 1 e 2). Essas condições favorecem a biossíntese de compostos secundários com propriedades antioxidantes (compostos fenólicos e carotenóides totais), como é apresentado na Tabela 3.

$\mathrm{Na}$ avaliação da atividade antioxidante do extrato aquoso e das frações de ácidos fenólicos da polpa do pequi pelos métodos de co-oxidação do b-caroteno ácido linoléico e teste de Rancimat, Lima \& Mancini-Filho (2005) evidenciaram proteção contra os danos oxidativos comparados ao padrão comercial butilidroxitolueno (BHT).

A polpa do pequi possui $209 \mathrm{mg} / 100 \mathrm{~g}$ de fenólicos totais, valores superiores aos encontrados na maioria das polpas de frutas consumidas no Brasil, como: Açaí (Euterpe oleracea), com 136,8mg/100g; goiaba (Psidium guayava), com 83,1mg/100g; morango (Gingo biloba), com 132,1mg/100g; abacaxi (Ananas sativa), com 21,7mg/100g; graviola (Anona muricato), com $84,3 \mathrm{mg} / 100 \mathrm{~g}$, e maracujá (Passiflora edulis), com 20,2mg/100g, sendo inferior apenas à acerola (Malpighia glabra), com $580,1 \mathrm{mg} / 100 \mathrm{~g}$, e à manga (Mangifera indica), com $544 \mathrm{mg} / 100 \mathrm{~g}$. Esses resultados indicam que a polpa do pequi é um alimento com elevada capacidade antioxidante, demonstrando a correlação existente entre a quantidade de fenólicos totais e a proteção antioxidante (Kuskoski et al., 2005).

A polpa do pequi possui $7,25 \mathrm{mg} / 100 \mathrm{~g}$ de carotenóides totais. De acordo com Godoy \& Rodrigues-Amaya (1994), os $\alpha$ e 
$\beta$ carotenos juntos representam $10 \%$ dos pigmentos carotenóides totais na polpa do pequi. Esses mesmos autores encontraram valores muito próximos aos detectados neste trabalho para os carotenóides totais $(7,46 \mathrm{mg} / 100 \mathrm{~g})$. Dentre os frutos do cerrado, o teor de carotenóides do pequi é superado apenas pela polpa de buriti, com $16,7 \mathrm{mg} / 100 \mathrm{~g}$.

A amêndoa do pequi possui um teor reduzido de fenólicos e de carotenóides, como mostra a Tabela 3. Esses valores estão próximos aos de outras amêndoas típicas do cerrado brasileiro, como o baru (Dipteryx alata), a bocaiúva (Calophyllum brasiliense) e o caju (Anarcadium occidentale) (Crepaldi et al., 2001).

Dessa forma, pode-se observar que, na polpa e na amêndoa do pequi, os lipídios são os constituintes predominantes, prevalecendo nestes os ácidos graxos oléico e palmítico. Na polpa, também se detectam um teor elevado de fibra alimentar e a presença de compostos fenólicos e carotenóides totais, os quais estão associados à prevenção de processos oxidativos.

TABELA 1 - Composição centesimal da polpa e amêndoa do pequi em base úmida.*

\begin{tabular}{lcc}
\hline Constituintes & Polpa & Amêndoa \\
\hline Umidade (\%) & $41,50 \pm 2,00$ & $8,68 \pm 0,08$ \\
Cinzas $(\mathrm{g})$ & $0,63 \pm 0,01$ & $4,01 \pm 0,51$ \\
Proteína(g) & $3,00 \pm 0,13$ & $25,27 \pm 0,74$ \\
Lipídios(g) & $33,40 \pm 3,76$ & $51,51 \pm 0,35$ \\
Carboidratos(g) & 11,45 & 8,33 \\
Fibra alimentar total(g) & $10,02 \pm 0,2$ & $2,20 \pm 0,1$ \\
\hline Valor Energético Total (Kcal) & 358,4 & 598,3 \\
\hline
\end{tabular}

* Valores expressos como média \pm desvio-padrão

TABELA 2 - Composição percentual de ácidos graxos da polpa e amêndoa de pequi.*

\begin{tabular}{lccc}
\hline \multicolumn{1}{c}{ Ácidos graxos } & $\mathbf{N}^{\circ}$ carbonos & Polpa & Amêndoa \\
\hline Láurico & $\mathrm{C} 12: 0$ & $0,04 \pm 0,00$ & $\mathrm{nd}$ \\
Mirístico & $\mathrm{C} 14: 0$ & $0,13 \pm 0,01$ & $0,46 \pm 0,01$ \\
Palmítico & $\mathrm{C} 16: 0$ & $35,17 \pm 0,27$ & $43,76 \pm 0,04$ \\
Palmitoléico & $\mathrm{C} 16: 1$ & $1,03 \pm 0,00$ & $1,23 \pm 0,03$ \\
Esteárico & $\mathrm{C} 18: 0$ & $2,25 \pm 0,04$ & $2,54 \pm 0,06$ \\
Oléico & $\mathrm{C} 18: 1 \mathrm{n}-9$ & $55,87 \pm 0,30$ & $43,59 \pm 0,16$ \\
Cis-vacênico & $\mathrm{C} 18: 1 \mathrm{n}-7$ & $1,90 \pm 0,08$ & $1,38 \pm 0,01$ \\
Linoléico & $\mathrm{C} 18: 2$ & $1,53 \pm 0,02$ & $5,51 \pm 0,08$ \\
$\alpha$-linolênico & $\mathrm{C} 18: 3$ & $0,45 \pm 0,00$ & $0,09 \pm 0,00$ \\
Araquídico & $\mathrm{C} 20: 0$ & $0,23 \pm 0,00$ & $0,20 \pm 0,00$ \\
Gadoléico & $\mathrm{C}: 20: 1$ & $0,27 \pm 0,01$ & $0,04 \pm 0,00$ \\
Docosaexaenóico & $\mathrm{C} 22: 6$ & $\mathrm{nd}$ & $0,19 \pm 0,02$ \\
\hline Total de saturados & & 37,97 & 47,17 \\
Total de insaturados & & 61,35 & 52,48 \\
Não identificados & & 0,68 & 0,35 \\
\hline
\end{tabular}

nd = não detectado

* Valores expressos como média \pm desvio-padrão

TABELA 3 - Teores de fenólicos totais e carotenóides totais em $\mathrm{mg} / 100 \mathrm{~g}$, na polpa e amêndoa de pequi.*

\begin{tabular}{ccc}
\hline Constituintes & Polpa & Amêndoa \\
\hline Fenólicos totais & $209,0 \pm 0,05$ & $122,0 \pm 0,05$ \\
Carotenóides totais & $7,25 \pm 0,6$ & $0,295 \pm 0,5$ \\
\hline
\end{tabular}

* Valores expressos como média \pm desvio-padrão 


\section{AGRADECIMENTOS}

Os autores agradecem ao CNPq, pelo apoio financeiro no desenvolvimento do trabalho e pela bolsa de estudos de Doutorado concedida ao primeiro autor.

\section{REFERÊNCIAS}

ALMEIDA, L.B.; PENTEADO, M.V.C. Carotenoids and provitamin A value of white fleshed Brasilian sweet potatoes (Ipomoea batatas Lam.) Journal of Food Composition and Analysis, Roma, v. 1, p.249-258, 1988.

ALMEIDA, S.P.; SILVA, J.A. Piqui e Buriti: importância alimentar para a população dos cerrados. Planaltina: EMBRAPA-CPAC, 1994.38p.

ASSOCIATION OF OFFICIAL ANALYTICAL CHEMISTS. Official methods of analysis of the Association of Official Analytical Chemists. $16^{\text {th }}$ ed. Arlington: AOAC, 1998. v.1

CREPALDI, I.C.; ALMEIDA-MURADIAN, L.B.; PENTEADO, M.D.C.; SALATINO, A. Composição nutricional do fruto de licuri (Syagrus coronata Martius Beccari). Revista Brasileira de Botânica, São Paulo, v. 24, n. 2, p. 155-159, 2001.

FOLCH, J.; LEES, M.; STANLEY, G.H.S. A simple method for the isolation and purification of total lipids. Journal of Biological Chemistry, Bethesda, v.226, p.497, 1957.

GODOY, H. T.; RODRIGUEZ-AMAYA, D. B. Occurrency of cisIsomers of provitamin A in brazillian fruits. Journal Agricultural Food Chemistry, Easton, v. 42, p. 1306-1313, 1994.

HARTMAN, L., LAGO, R.C.A. Rapid preparation of fatty acids methyl esters. Laboratory Practice, London, v.22, p.475-476, 1973.

HINNEBURG, I; DAMIEN,H.J; RAIMO H. Antioxidant activities of extracts from selected culinary herbs and spices. Food Chemistry, London, v. 97, n. 1, p. 122-129, 2006.
KUSKOSKI, E.M.; ASUERO, G.A.; TRONCOSO, A.M.; MANCINI-FILHO, J.; FETT, R. Aplicación de diversos métodos químicos para determinar actividad antioxidante em pulpa de frutos. Revista de Ciência e Tecnologia de Alimentos, Campinas, v.25, n.4, p.726-732, 2005.

LIMA, A.; MANCINI-FILHO, J. Compostos com atividade antioxidante no fruto Pequi (Caryocar brasiliense, L), Revista da Sociedade Brasileira de Alimentação e Nutrição (NUTRIRE), São Paulo, v. 30, p. 310, 2005.

MICHELS, K.B.; FUCHS, C.S.; GIOVANNUCCI, E.; COLDIT, A.; HUNTER, D. J.; STAMPFER, M.J.;.WILLETT, W.C.; Fiber intake and incidence of colorectal cancer among 76,947 women and 47,279 men. Cancer Epidemiology Biomarkers \& Prevention, Philadelphia, v.14, p.842-849,2005.

MELO JUNIOR, A.F.; CARVALHO, D.; PÓVOA, J.S.R.; BEARZOTI, E. Estrutura genética de populações naturais de pequizeiro (Caryocar brasiliense Camb.). Scientia Forestalis, Piracicaba, n. 66, p.56-65, 2004.

NÚCLEO DE ESTUDOS E PESQUISAS EM ALIMENTAÇÃO. Tabela brasileira de composição de alimentos. Campinas: NEPA/ UNICAMP, 2004. 42p.

RIBEIRO, R.F. Pequi: o rei do cerrado. Belo Horizonte: Rede Cerrado, 2000. 62p.

RODRIGUES-AMAYA, D.B. Carotenoids and food preparation: the retention of provitamin A carotenoids in prepared, processed, and stored foods. Arlington: OMNI project, 1997. 88p.

WAIN, T.; HILLS, W.E. The phenolic constituents of Punnus domestica. I-quantitative analysis of phenolic constituents. Journal Science of. Food Agricutural, London, v.19, p.63-68, 1995. 